\title{
Immunochemistry of the Surface Carbohydrate Antigens of Bacteroides fragilis and Definition of a Common Antigen
}

\author{
By IAN R. POXTON* AND ROBERT BROWN \\ Bacteriology Department, University of Edinburgh Medical School, Teviot Place, \\ Edinburgh EH8 $9 A G, U K$
}

(Received 7 February 1986; revised 10 April 1986)

\begin{abstract}
The components extracted by aqueous phenol from whole cells of Bacteroides fragilis were analysed by SDS-PAGE and immunoblotting and shown to consist of a series of strain-specific, cross-reactive and common antigens. Regularly-spaced ladder patterns on silver-stained gels indicated that in most strains the LPS was present as a predominantly smooth type, but with chain lengths of varying molecular mass, ranging within each particular strain from essentially rough forms to long chain-length smooth forms. The rough form of the LPS at the gel front possessed an antigen common to most of the strains investigated. Another antigen, which migrated behind the rough LPS on SDS gels, was common to all strains of the species. The smooth LPS forms and the other high molecular mass components were strain-specific antigens. Previously published methods are not capable of producing pure LPS or capsular polysaccharide for this organism.
\end{abstract}

\section{INTRODUCTION}

Bacteroides fragilis, the most frequently isolated anaerobic bacterium from clinical specimens, appears to have a greater pathogenic potential than its close relatives, e.g. B. vulgatus and other members of the $B$. fragilis group. Surface structures have been associated with this virulence (Onderdonk et al., 1977). Much has been published on the chemistry and immunochemistry of structural components but confusion exists. Many workers (e.g. Kasper et al., 1983) assume that the surface carbohydrates of this Gram-negative bacterium consist simply of lipopolysaccharide (LPS) and 'capsular polysaccharide'. Hofstad (1981) and Cousland \& Poxton (1984), however, have demonstrated a complex set of surface carbohydrates. Much of the apparent confusion stems from the difficulty in preparing pure surface components. Kasper et al. (1983) and Weintraub et al. (1985) claimed to have developed methods for the preparation of pure capsular polysaccharide free from LPS, and of LPS free from capsular polysaccharide, protein and nucleic acid. Starting with an aqueous phenol extract of whole cells, capsular polysaccharide was prepared by gel filtration in the presence of a detergent, whereas LPS was prepared by further extraction with a phenol/chloroform/petroleum spirit mixture. The authors suggested that the LPS of $B$. fragilis is a rough-type molecule and is both chemically and antigenically similar in all the strains investigated.

The development of a method for demonstrating the heterogeneity of LPS on polyacrylamide gels (Tsai \& Frasch, 1982) has been applied to many species of Gram-negative bacteria. In a preliminary report (Cousland \& Poxton, 1983), we showed that $B$. fragilis possesses a complex mixture of aqueous phenol-extractable surface carbohydrate antigens. A series of closely spaced bands in a ladder pattern on the silver-stained polyacrylamide gel was reminiscent of the pattern produced by the smooth form of LPS from other bacteria. This led us to suggest that $B$. fragilis possesses smooth type LPS. We also showed that a common antigen ran at the gel front and was associated in some unknown way with the rough form of the molecule. 
In the present paper we extend our earlier work by studying aqueous phenol extracts of a range of $B$. fragilis strains with a combination of SDS-PAGE and immunoblotting. We report on the complexity of the carbohydrates of the cell-surface of $B$. fragilis and we define the common antigen.

\section{METHODS}

Bacterial strains and growth conditions. B. fragilis strains NCTC 9343 and 9344 were obtained from the National Collection of Type Cultures, Colindale Avenue, London, UK. Clinical strains of $B$. fragilis, GNAB 4, 82, 85, 90 and 92 , were from our laboratory collection.

All strains were cultured in 2 to 6 litre batches of proteose peptone/yeast extract (PPY) medium (Deacon et al., 1978 ) statically for $18 \mathrm{~h}$ in an anaerobic atmosphere $\left(80 \% \mathrm{~N}_{2}, 10 \% \mathrm{CO}_{2}\right.$ and $\left.10 \% \mathrm{H}_{2}\right)$ at $37{ }^{\circ} \mathrm{C}$.

Preparation of aqueous phenol extract. Lyophilized bacteria were resuspended to a concentration of approximately $5 \%(\mathrm{w} / \mathrm{v})$ in distilled water and heated to $67^{\circ} \mathrm{C}$. An equal volume of $90 \%(\mathrm{w} / \mathrm{w})$ aqueous phenol at $67^{\circ} \mathrm{C}$ was added to the suspension and the mixture was stirred for $15 \mathrm{~min}$ at $67^{\circ} \mathrm{C}$. After cooling to $4{ }^{\circ} \mathrm{C}$ the phases were separated by centrifugation at $10000 \mathrm{~g}$ for $10 \mathrm{~min}$. The upper aqueous phase was dialysed against running water for $18 \mathrm{~h}$, concentrated to approximately $20 \%$ of the original volume by rotary evaporation and centrifuged at $100000 \mathrm{~g}$ for $3 \mathrm{~h}$ to sediment the insoluble fraction. This was washed once by suspension in water with the aid of a syringe fitted with a no. 26 gauge needle, and recentrifugation. The pellet was finally resuspended in water and lyophilized.

Fractionation of aqueous phenol extract. The lyophilized pellet from the aqueous phenol extract $(5 \mathrm{mg}) \mathrm{was}$ solubilized in deoxycholate buffer and fractionated on a column $(55 \times 1.6 \mathrm{~cm})$ of Sephacryl S-300 by the method of Kasper et al. (1983). Fractions $(50 \times 2 \mathrm{ml})$ were analysed for precipitating antigen by fused rocket immunoelectrophoresis in the presence of $1 \%$ Triton X-100 (Svendsen, 1973), and for total antigen by dot-blotting onto nitrocellulose membrane and reaction with antiserum to strains of $\boldsymbol{B}$. fragilis as described below.

Preparation of rough LPS. Lyophilized, dialysed aqueous phase from the aqueous phenol extraction of $B$. fragilis NCTC 9344 was extracted with phenol/chloroform/petroleum by the method used for LPS extraction by Weintraub et al. (1985). The material remaining insoluble after phenol/chloroform/petroleum extraction was resuspended in distilled water, re-extracted with an equal volume of $90 \%$ aqueous phenol as described above and the aqueous phase dialysed and lyophilized.

Preparation of antisera. Antisera were raised in New Zealand White rabbits to whole, live, washed cells of $B$. fragilis strains NCTC 9344, GNAB 4 and GNAB 92 by the method of Poxton (1979).

Monospecific polyclonal antiserum was raised to the $B$. fragilis common antigen by a method briefly referred to in a Bio-Rad pamphlet (no. 48EG, 1984). The aqueous phenol extract (containing $112 \mu \mathrm{g}$ carbohydrate) was separated as a band on SDS-PAGE and transferred to nitrocellulose as described below. This was done in duplicate. Guide strips were cut from the nitrocellulose and reacted with whole cell antiserum to locate the common antigen. Strips $2 \mathrm{~mm}$ wide containing the common antigen were cut from the unreacted nitrocellulose membranes. They were dried in a stream of compressed air, cut into small pieces and dissolved in dimethyl sulphoxide $\left(100 \mu \mathrm{cm}^{-2}\right)$. This was mixed with an equal volume of Freund's complete adjuvant (Difco) and injected subcutaneously in approximate $0.1 \mathrm{ml}$ volumes into several sites on the back of a rabbit. This procedure was repeated four times but with Freund's incomplete adjuvant over a period of two months. Test bleedings taken at intervals during the injection schedule were screened by dot-blotting against homologous aqueous phenol extract. The antiserum reached its highest titre one month after the last injection.

SDS-PAGE. This was done on both $10 \%$ and $12 \%$ slab gels with the buffer system of Laemmli (1970) by the method described by Poxton \& Brown (1979). Gels were oxidized with periodate and stained with silver by the method of Tsai \& Frasch (1982).

Electrophoretic transfer and immunoblot. This was done by the method described by Cousland \& Poxton (1983), with the Bio-Rad Immunoblot reagents, except that the nitrocellulose membrane was $0.2 \mu \mathrm{m}$ pore size (Sartorius). Antigens were either electrophoretically transferred, or applied directly in $1 \mu$ l volumes as dots for dot-blotting. The antiserum raised against whole cells was used at a dilution of 1 in 250 while that raised against the common antigen was used at a dilution of 1 in 40.

Analytical methods. Carbohydrate was measured by the method of Dubois et al. (1956) with glucose as a standard. Protein was measured by the Lowry method.

\section{RESULTS}

The bacteria grew well in PPY medium to yield $0.5-1.0 \mathrm{~g}$ dry weight $\mathrm{l}^{-1}$ after overnight incubation. After aqueous phenol extraction and ultracentrifugation, yields of extract were $0.4 \%$ to $0.9 \%$ of the bacterial dry weight. All strains were grown on more than one occasion; yields were similar and subsequent analyses were identical. 


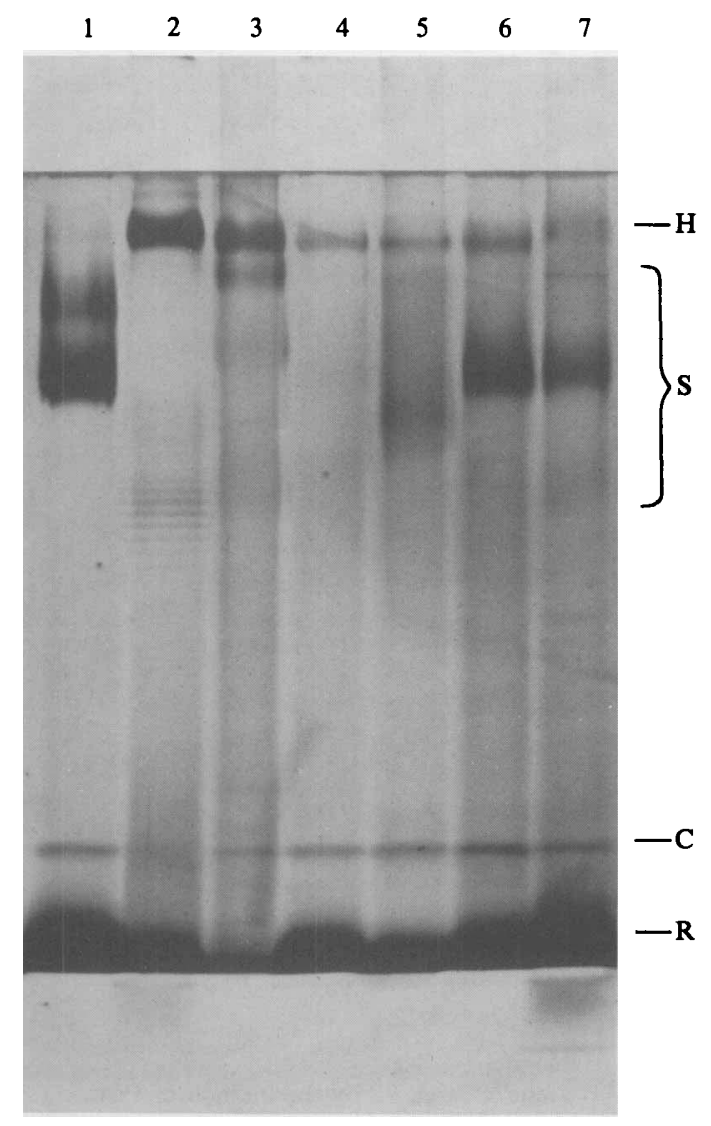

Fig. 1. Silver-stained SDS-polyacrylamide ( $12 \%)$ gels of aqueous phenol extracts ( $10 \mu \mathrm{g}$ carbohydrate) of seven strains of $B$. fragilis. Track 1, NCTC 9343; track 2, NCTC 9344; track 3, GNAB 4; track 4, GNAB 82; track 5, GNAB 85; track 6, GNAB 90; track 7, GNAB 92. H, High molecular mass band; $S$, multi-banded pattern typical of smooth LPS; C, common antigen; $R$, rough LPS at gel front.

Samples of dry extract were resuspended in distilled water to a concentration of $5 \mathrm{mg} \mathrm{ml}^{-1}$ and assayed for carbohydrate and protein. There was a negligible amount of protein, and phenol/sulphuric acid positive carbohydrate (Dubois et al., 1956) accounted for about $20 \%$ only of the dry weight of the extract. No further chemical assays were done.

\section{SDS-PAGE and immunoblotting of aqueous phenol extracts}

Each extract was dissolved to a concentration of $400 \mu \mathrm{g}$ carbohydrate $\mathrm{ml}^{-1}$ in the SDS/2mercaptoethanol solubilization buffer of Laemmli (1970), and heated to $100^{\circ} \mathrm{C}$ for $3 \mathrm{~min}$. Samples $(25 \mu \mathrm{l})$ were applied to a $12 \%$ SDS-polyacrylamide gel. Fig. 1 shows examples of patterns obtained after separation and silver staining of aqueous phenol extracts from seven strains of $B$. fragilis. In all tracks there is a multi-banded pattern (labelled $S$ ), of varying intensity, which is similar to smooth LPS of other Gram-negative bacteria. In some tracks $(3,4$ and 5) the fine banding pattern is extremely faint and the general appearance is more like that of rough LPS. There is material of high molecular mass with a characteristic dumb-bell shape in most of the tracks (labelled $\mathrm{H}$ ). A pronounced band $(\mathrm{C})$ is present behind the main front band (R). No Coomassie-blue-staining material was observed.

Three separations identical to that shown in Fig. 1 were done, and the components from each were transferred to a sheet of nitrocellulose and probed with antisera raised to whole cells of 


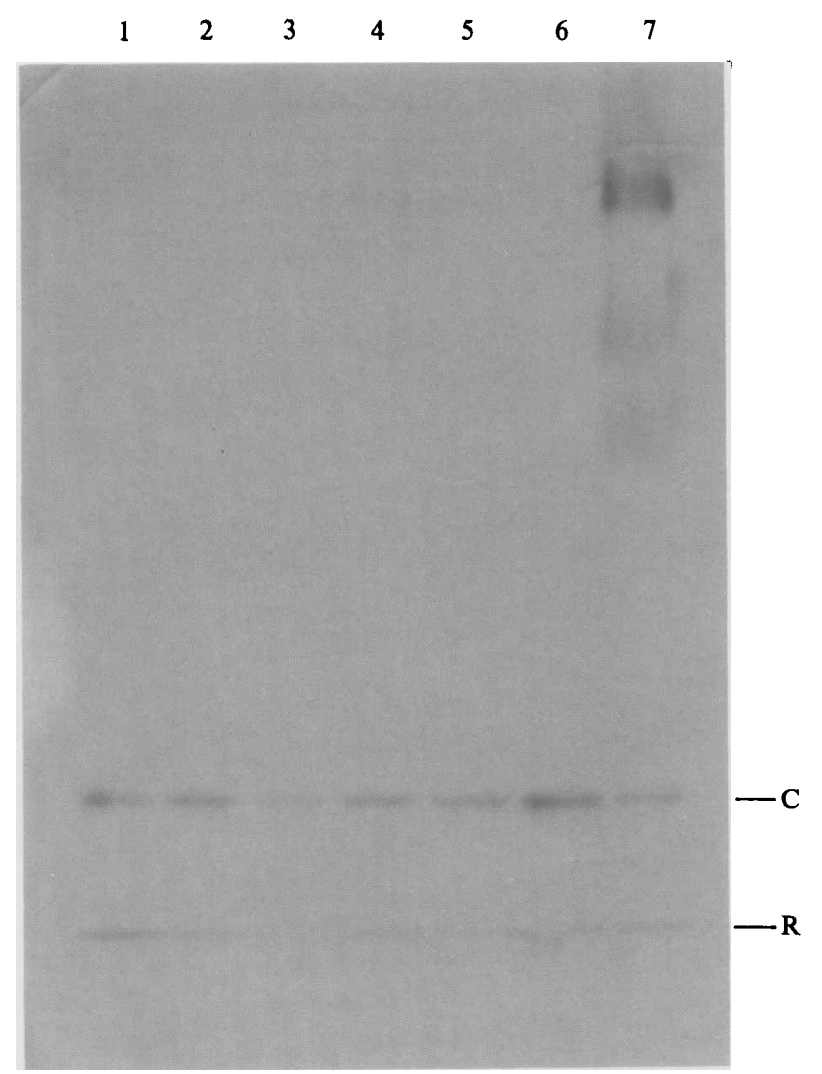

Fig. 2. Immunoblots from SDS-polyacrylamide $(12 \%)$ gels of aqueous phenol extracts of the strains used in Fig. 1. The blots were probed with antiserum raised against whole cells of $\boldsymbol{B}$. fragilis GNAB 92 and anti-rabbit horseradish peroxidase conjugate. See Fig. 1 for abbreviations.

three of the strains: NCTC 9344, GNAB 4 and GNAB 92. An example of the results (with GNAB 92 antiserum) is shown in Fig. 2. All strains have one antigen in common, a discrete band migrating behind the front (labelled $C$ ). The band migrating at the front $(R)$ is an antigen common to all strains except GNAB 4, whereas antigens in the upper part of the gel were detected only in the homologous strain (track 7) and weakly in strain GNAB 82 (track 4). With the NCTC 9344 antiserum, a similar pattern was seen: an antigen common to all strains was detected just behind the gel front, a band at the gel front was common to all except GNAB 4 and a series of higher molecular mass bands were common to the homologous strain, NCTC 9343 and GNAB 4. With the GNAB 4 antiserum, the common band behind the gel front was apparent, and a series of higher molecular mass bands common to the same three strains as with the NCTC 9344 were visible, but the band at the front was specific only to the homologous reaction. Pre-immune sera gave no reactions.

\section{Fractionation of aqueous phenol extract}

As the aqueous phenol extract consisted of a series of different antigens demonstrable by SDSPAGE and immunoblotting, the whole extract was subjected to gel filtration in the presence of deoxycholate by the method developed by Kasper et al. (1983) for the production of capsular polysaccharide free from LPS. The extract from $B$. fragilis GNAB 92 was solubilized and fractionated on Sephacryl S-300. Analysis of the fractions by fused rocket immunoelectrophoresis with homologous whole cell antiserum showed that only fractions eluted at the void volume of the column contained precipitating antigens. When the fractions were analysed by dot blotting, 


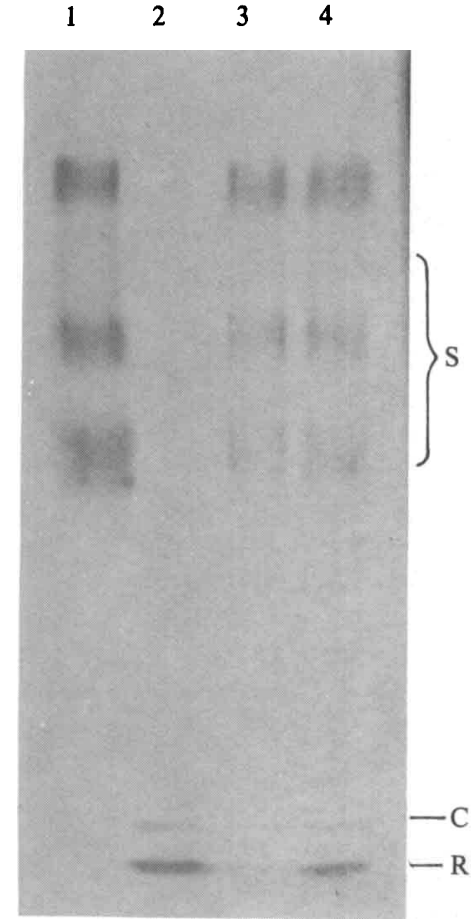

Fig. 3
1

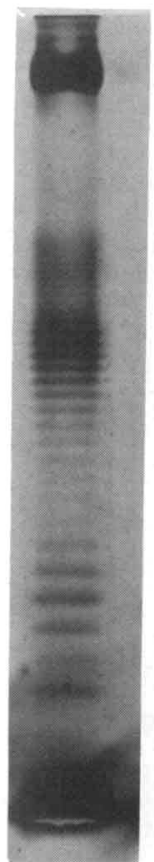

2

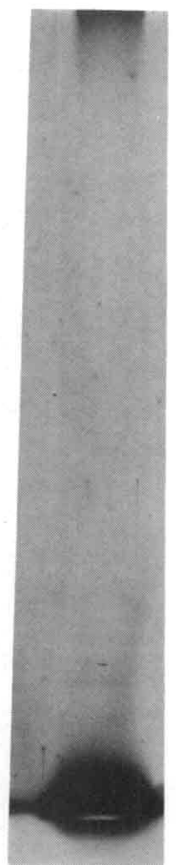

Fig. 4
3

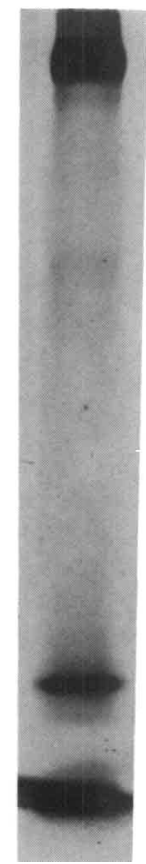

Fig. 3. Immunoblots from SDS-polyacrylamide (10\%) gels of an aqueous phenol extract of $B$. fragilis GNAB 92 after fractionation on Sephacryl S-300. Track 1, pooled void volume antigenic fractions; track 2, pooled included antigenic fractions; track 3, fractions from between two antigenic peaks; track 4, unfractionated aqueous phenol extract. All tracks contained $10 \mu \mathrm{g}$ carbohydrate. The blots were probed with homologous whole cell antiserum and horseradish peroxidase conjugate. See Fig. 1 for abbreviations.

Fig. 4. Silver-stained SDS-polyacrylamide (12\%) gels of extracts $(10 \mu \mathrm{g}$ carbohydrate) of $\boldsymbol{B}$. fragilis NCTC 9344. Track 1, aqueous phenol extract; track 2, phenol/chloroform/petroleum extract of aqueous phenol extract; track 3, material remaining insoluble after phenol/chloroform/petroleum extraction.

two groups of antigen-containing fractions were detected, one corresponding to the column void volume which was the same as that detected by fused rocket immunoelectrophoresis and one containing lower molecular mass material. With the two other antisera (GNAB 4 and NCTC 9344), only the lower molecular mass material reacted. These findings indicate that the first peak contained strain-specific antigens while the second contained cross-reactive antigens.

The fractions that corresponded to each of the two peaks of antigen as demonstrated by dot blotting, and the fractions between the two peaks, were combined into three pools, lyophilized and examined by SDS-PAGE and immunoblotting; both techniques gave identical results. The patterns produced with homologous antiserum are shown in Fig. 3. The void volume fractions (track 1) contained a series of antigens of which the central group (labelled S), on close examination of the original, consisted of a series of discrete fine bands. The included fractions (track 2) produced only two bands corresponding to the common $B$. fragilis antigen (C), and the front band (R). The pooled fractions between the peaks (track 3) contained a mixture of the antigens in both of the peaks. Track 4 is of the aqueous phenol extract before fractionation; fractionation of the aqueous phenol extract of GNAB 4 gave similar results. 


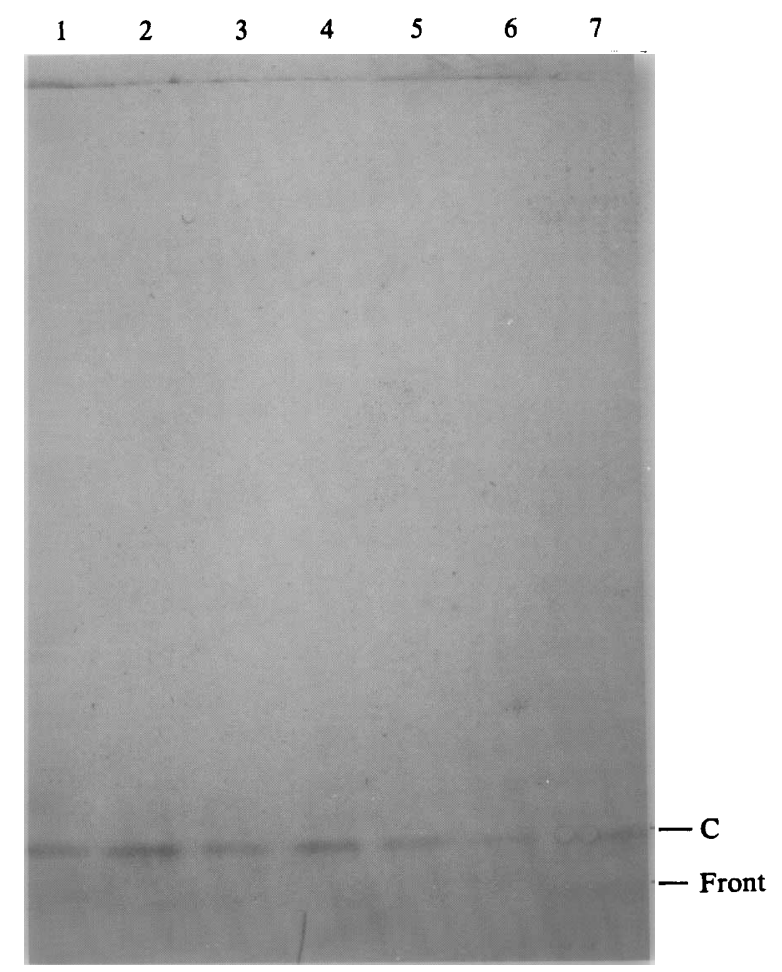

Fig. 5. Immunoblots from SDS-polyacrylamide (12\%) gels of aqueous phenol extracts of the strains used in Fig. 1. The blots were probed with monospecific antiserum raised against the common antigen excised from nitrocellulose.

\section{Comparison of rough LPS with aqueous phenol extract}

The pattern produced on SDS-PAGE of the phenol/chloroform/petroleum-extracted fraction of the aqueous phenol extract of NCTC 9344, i.e. the fraction corresponding to the LPS studied by Weintraub et al. (1985), was compared with that of the aqueous phenol extract and that of the material that remained insoluble after the phenol/chloroform/petroleum treatment (Fig. 4). A ladder pattern typical of smooth LPS was found with the aqueous phenol extract, with the dumbbell shaped high molecular mass band. Track 2 shows the PCP fraction, which was typical of rough LPS, with only a single heavily staining band at the front. Track 3 , containing the insoluble material remaining after PCP treatment, had a strongly staining dumb-bell shaped band at the top, a faintly staining ladder pattern that became stronger after longer development, an intensely stained band running behind the front (subsequently shown by blotting to be the common antigen) and also a band at the dye front.

\section{Demonstration of the antigenic specificity of the $B$. fragilis common antigen}

Common antigen, purified by SDS-PAGE, immunoblotting and extraction from nitrocellulose membranes, was injected into a rabbit to raise monospecific antiserum. In immunoblots against aqueous phenol extracts prepared from the seven strains used in Figs 1 and 2, the serum reacted strongly with the common band of the $B$. fragilis strains (Fig. 5).

\section{DISCUSSION}

In this study we have shown that aqueous phenol extracts of $B$. fragilis contain a series of antigens that can be detected by silver-staining and immunoblotting. Most strains possess antigens that include (i) a rough type LPS which runs at the gel front, (ii) a common antigen which runs behind the front, (iii) a smooth LPS which runs as a series of closely spaced bands, 
and (iv) one other antigen, with a high molecular mass and with a characteristic dumb-bell pattern, which we suggest is the capsular polysaccharide.

The recent studies of Weintraub et al. (1985) on the chemistry and immunochemistry of the LPS of $B$. fragilis were based on the assumption (shown here to be false) that the species produced only a rough form of LPS; the system used selectively extracted rough LPS and the fraction that would have contained the smooth form was discarded. In the earlier studies of these workers (Kasper et al., 1983), the Sephacryl S-300 chromatography separated the aqueous phenol extract into two major fractions; the higher molecular mass fraction consisted of 'capsular polysaccharide', while the second fraction contained LPS. In our studies, the first peak consisted of smooth LPS together with another high molecular mass antigen, probably capsular polysaccharide, and the second peak contained rough LPS and the common antigen.

The nature of the common antigen is not known. The monospecific antiserum raised against it shows that it is antigenically distinct from the rough form of the LPS, although in some immunoblots a weak reaction was seen with some of the front material. Studies are currently in progress to purify and characterize the antigen with monoclonal antibodies, but at present we can only speculate on its nature. It may either be totally distinct from the LPS and be analogous to the enterobacterial common antigen (Mannel \& Mayer, 1978), as it is purified in a similar way, or be related to the rough LPS but with a unique and dominant epitope: it could be lipid A plus a complete core - the unique epitope, distal from the lipid end, being normally substituted with $\mathrm{O}$ antigen. The material that runs at the gel front, which is common to all but one of the strains of $\boldsymbol{B}$. fragilis that we have investigated, may be lipid A with an incomplete core. Its epitopes would be expressed along the linear core portion of all or most LPS molecules. The smooth LPS and the high molecular mass 'capsular' antigen are not common antigens. There is a certain amount of cross-reaction between the strains of the species but there appear to be typespecific antigens which could be used in serotyping.

This study was funded by a grant from the Medical Research Council. We are grateful to J. Keen for producing Fig. 3, C. Patrizio for expert technical assistance and to Professor J. G. Collee for encouragement and interest.

\section{REFERENCES}

Cousland, G. \& Poxton, I. R. (1983). Analysis of lipopolysaccharides of Bacteroides fragilis by sodium dodecyl sulphate-polyacrylamide gel electrophoresis and electroblot transfer. FEMS Microbiology Letters 20, 461-465.

Cousland, G. \& Poxton, I. R. (1984). Crossed immunoelectrophoresis and enzyme-linked immunosorbent assay of the cell-surface antigens of Bacteroides fragilis. Journal of General Microbiology 130, 645-655.

Deacon, A. G., Duerden, B. I. \& Holbrook, W. P. (1978). Gas-liquid chromatographic analysis of metabolic products in the identification of Bacteroidaceae of clinical interest. Journal of Medical Microbiology 11, 81-99.

Dubois, M., Gilles, K. A., Hamilton, J. K., Rebers, P. A. \& SMITH, F. (1956). Colorimetric method for determination of sugars and related substances. Analytical Chemistry 28, 350-356.

HofstaD, T. (1981). Precipitating carbohydrate antigens of Bacteroides fragilis NCTC 9343. Acta pathologica et microbiologica scandinavica B89, 215219.

KaSPer, D. L., Weintraub, A., Lindberg, A. A. \& LONNGREN, J. (1983). Capsular polysaccharide and lipopolysaccharide from two Bacteroides fragilis reference strains: chemical and immunochemical characterisation. Journal of Bacteriology 153, 991997.

LAEMMLI, U. K. (1970). Cleavage of structural proteins during the assembly of the head of bacteriophage T4. Nature, London 227, 680-685.

MANNEL, D. \& MAYER, H. (1978). Isolation and chemical characterisation of the enterobacterial antigen. European Journal of Biochemistry 86, 361370.

Onderdonk, A. B., Kasper, D. L., Cisneros, R. L. \& BARTLETT, J. G. (1977). The capsular polysaccharide of Bacteroides fragilis as a virulence factor: comparison of the pathogenic potential of encapsulated and unencapsulated strains. Journal of Infectious Diseases 136, 82-89.

Poxton, I. R. (1979). Serological identification of Bacteroides species by an enzyme-linked immunosorbent assay. Journal of Clinical Pathology 32, 294-298.

Poxton, I. R. \& BRown, R. (1979). Sodium dodecyl sulphate-polyacrylamide gel electrophoresis of cellsurface proteins as a guide to the identification of the Bacteroides fragilis group. Journal of General Microbiology 112, 211-217.

SVENDSEN, P. J. (1973). Fused rocket immunoelectrophoresis. Scandinavian Journal of Immunology 2, supplement 1, 69-70.

TsaI, C.-M. \& Frasch, C. E. (1982). A sensitive silver stain for detecting lipopolysaccharides in polyacrylamide gels. Analytical Biochemistry 119, 115-119.

Weintraub, A., Larsson, B. E. \& Lindberg, A. A. (1985). Chemical and immunochemical analyses of Bacteroides fragilis lipopolysaccharides. Infection and Immunity 49, 197-201. 